\title{
MODELAGEM DINÂMICA E CONTROLE DO TCSC PARA APLICAÇÕES EM SISTEMAS DE DISTRIBUIÇÃO DE ENERGIA ELÉTRICA
}

\author{
LuCiano S. C. E Silva*, LeONARdo ZAMPEllin , Dionízio PASChOAREli JR ., FALCONDES J. M. DE SEIXAS, \\ PATRICK R. DE AlMEIDA, Wilson M. BORGES, HENRIQUe L. GARCIA, JosÉ F. RESENDE.
}

\author{
Lab. de Eletrônica de Potência, Dep. de Engenharia Elétrica, Univ. Estadual Paulista - UNESP \\ Caixa Postal 31, 15385-000- Ilha Solteira, SP, Brasil \\ *IFG-Instituto Federal de Educação, Ciência e Tecnologia de Goiás \\ Departamento das Áreas Acadêmicas de Jataí \\ Rua Riachuelo, 2090, 75804-020, Jataí, Goiás, Brasil \\ E-mails: lucianocosta_@hotmail.com, leozampellin@ig.com.br, \\ dionizio@dee.feis.unesp.br, falcon@dee.feis.unesp.br, \\ patrick_roberto@hotmail.com, Wilson.Borgesdelektro.com.br, \\ Henrique.Garcia@elektro.com.br, Jose.Resende@elektro.com.br
}

\begin{abstract}
This paper proposes a closed-loop control of a Thyristor Controlled Series Compensator (TCSC) for application on distribution systems. Using the linear fundamental frequency model of the TCSC was proposed a control system in d-q coordinates, with orientation on the capacitor voltage, which answer the request of reactance with good transient response and provides rejection to disturbances and oscillations of distribution system. The simulation results obtained demonstrate the proper operation of the control system for the tests step response reference and load variations. These results will subsidize the construction of an experimental prototype.
\end{abstract}

Keywords— TCSC, Distribution System, d-q control, Feedback Systems

Resumo- Este artigo propõe o controle em malha fechada de um Thyristor Controlled Series Compensator (TCSC) para aplicação em sistemas de distribuição. Utilizando um modelo linear na frequência fundamental do TCSC foi proposto um sistema de controle em coordenadas d-q, com orientação na tensão do capacitor, que atenda a solicitação de reatância com boa resposta transitória e proporcione rejeição às perturbações e oscilações do sistema de distribuição. Os resultados de simulação obtidos demonstram o funcionamento adequado do sistema de controle para a resposta em degrau na referência e variações de carga. Tais resultados subsidiarão a construção de um protótipo experimental.

Palavras-chave— TCSC, Sistema de Distribuição, Controle d-q, Sistemas realimentados

\section{Introdução}

Em sistemas de distribuição a resistência dos cabos da linha exerce forte influência na composição da impedância, podendo ser até maior que a reatância indutiva, dependendo do tipo de cabo utilizado. Deste modo, nas linhas de distribuição, as quais apresentam níveis de tensão na faixa de $13,8 \mathrm{kV}$, torna-se inerente o uso de bancos de capacitores e reguladores de tensão para se atender os consumidores com qualidade de energia à medida que o comprimento da linha aumenta. Equipamentos convencionais utilizados para elevar e regular o nível de tensão, como bancos de capacitores e reguladores de tensão, algumas vezes não são suficientemente eficientes. É o que ocorre em casos como os encontrados em linhas extensas com carga concentrada na sua extremidade, ou nas partidas de grandes motores de indução, pois o tempo de partida destes é menor que o tempo de resposta dos equipamentos convencionais. Isto exige que a concessionária insira no seu programa de expansão a construção de subestações, ramais de transmissão, alimentadores, elevando assim os custos dos investimentos (Marafão et al., 1994).

Os circuitos em uma rede de distribuição normalmente operam de forma radial, o que torna possí- vel a inserção do TCSC (Thyristor Controlled Series Compensator) em um desses circuitos para promover a compensação série e melhorar a qualidade da energia elétrica. A utilização da compensação série eletronicamente controlada permite variação rápida da reatância equivalente da linha, proporcionando rejeição às perturbações e oscilações indesejadas (Pilotto et al., 2003; Kabiri et al., 2004). No entanto, embora haja uma variedade de estudos e modelos dinâmicos sobre a operação do TCSC em sistemas de transmissão (Jalali et al., 1996; Xiaobo et al., 1998; FuerteEsquivel et al., 2000; Meikandasivam et al., 2010), sua aplicação no sistema de distribuição é novidade. Como os parâmetros da linha de distribuição diferem em relação à linha de transmissão, e devido à facilidade da inserção de compensadores auxiliares na linha, é importante avaliar o comportamento deste tipo de compensação controlada nos sistemas de distribuição.

Neste trabalho foi proposto um sistema de controle do TCSC em coordenadas d-q, com orientação na tensão do capacitor, que atenda a solicitação de reatância com boa resposta transitória e proporcione rejeição às perturbações e oscilações a que o sistema de distribuição está sujeito. Dentre os modelos propostos na literatura, utilizou-se para o projeto do controlador um modelo linear na frequência fundamental (Jovcic et al., 2005). 


\section{Sistema de Controle do TCSC aplicado em um Sistema de Distribuição}

A Figura 1 apresenta o esquema elétrico do compensador série TCSC aplicado em uma linha de distribuição. A compensação série, nesse tipo de aplicação, tem como principal função o suporte de tensão no ponto de acoplamento comum (PAC) considerando os transitórios de carga existentes no sistema. Como ilustrado na Figura 1, tal compensação é realizada eletronicamente via controle dos ângulos de disparo dos tiristores. Um sistema de controle eficaz, além de fornecer total sintonia da reatância série desejada, deve também proporcionar rejeição aos distúrbios provenientes de transitórios de carga ou resultantes de interações eletromecânicas no sistema, tais como partidas de grandes máquinas e possíveis ressonâncias subsíncronas. Assim, para o projeto de controladores, faz-se necessário o conhecimento de modelos dinâmicos que descrevam com relativa precisão tais comportamentos em determinadas faixas de frequência.

O sistema de controle proposto é realizado considerando o sistema de coordenadas d-q com orientação na tensão do capacitor do TCSC. Tal sistema é detalhado na Figura 2 e consiste basicamente de três etapas: transformação de referência de coordenadas e filtragem, malha de controle da reatância equivalente do TCSC e sincronização para o disparo dos tiristores. 
$g$ - função sinal (0 para tiristor bloqueado e 1 para tiristor em condução).

A tensão no capacitor pode ser expressa em duas parcelas: parcela controlável e não linear $\left(\mathrm{v}_{2}\right)$ e parcela não controlável e linear $\left(\mathrm{v}_{1}\right)$. Além disso, presume-se que a corrente de linha é constante em um ciclo fundamental, ou seja, só serão admitidas variações em frequências abaixo da componente fundamental. A tensão no capacitor e suas parcelas são indicadas de (3) a (5).

$$
\begin{gathered}
v_{1}=\frac{1}{C} \cdot \int i \cdot d t \\
v_{2}=\frac{1}{C} \cdot \int g \cdot i_{R C T} \cdot d t \\
v_{c}=v_{1}-v_{2}
\end{gathered}
$$

Após processo de investigação via simulação computacional encontra-se a resposta em frequência das componentes fundamentais da parcela não linear da tensão no capacitor. O modelo resultante representa a resposta dinâmica das componentes fundamentais das variáveis elétricas linearizadas em torno de um ponto de operação. Tal comportamento é apresentado em (6).

$$
\frac{v_{2}(s)}{v_{1}(s)}=\frac{\frac{s^{2}}{\omega_{n}^{2}(\alpha)}+\frac{2 \cdot \xi_{n}(\alpha) \cdot s}{\omega_{n}(\alpha)}+1}{\frac{s^{2}}{\omega_{d}{ }^{2}(\alpha)}+\frac{2 . \xi_{d}(\alpha) s}{\omega_{d}(\alpha)}+1}
$$

A equação (6) indica que o modelo é de segunda ordem com duplos polos e duplos zeros. No entanto, a característica dinâmica do sistema varia em função do ponto de operação. Os coeficientes de (6) são definidos em função do ângulo de disparo $\alpha$ nas equações de (7) a (10).

$$
\begin{gathered}
\omega_{d}=\frac{\pi-2 \cdot \alpha}{\pi \cdot \sqrt{L_{t c r} \cdot C}} \\
\xi_{d}=0,38 \cdot \cos (\alpha) \\
\omega_{n}=\pi \cdot f_{1} \cdot 10^{-3} \cdot\left(1,7+\sqrt{10} \cdot \frac{\alpha}{\tan ^{4}(\alpha)}\right) \\
\xi_{n}=0,20 \cdot \cos (\alpha)
\end{gathered}
$$

Para transferir o sistema para a representação em espaço de estados, definem-se as variáveis de estados em (11) a (14).

$$
\begin{gathered}
x_{2}=\frac{x_{1}=v_{1}}{\frac{s^{2}}{\omega_{d}^{2}}+\frac{2 . \xi_{d} s}{\omega_{d}}+1} \\
x_{3}=s \cdot x_{2} \\
x_{4}=\frac{v_{c}}{t_{f} \cdot s+1}
\end{gathered}
$$

Note que, se $\mathrm{t}_{\mathrm{f}}$ for muito pequeno, $\mathrm{x}_{4}$ representa a tensão no capacitor e não introduz dinâmica no sistema. Tal operação foi realizada somente para permitir a representação da tensão no capacitor como uma variável de estado. A corrente de linha é considerada uma entrada no sistema.

$$
\mathbf{u}=i
$$

Aplicando a transformada inversa de Laplace e rearranjando as equações de (11) a (14), chega-se na representação em espaço de estados em (16).

$$
\dot{\mathbf{x}}=\mathbf{A} \cdot \mathbf{x}+\mathbf{B}_{1} \cdot \mathbf{u}
$$

As matrizes de coeficientes são apresentadas em (17) e (18).

$$
\begin{gathered}
\mathbf{A}=\left[\begin{array}{cccc}
0 & 0 & 0 & 0 \\
0 & 0 & 1 & 0 \\
\omega_{d}^{2} & -\omega_{d}^{2} & -2 \cdot \xi_{d} \cdot \omega_{d} & 0 \\
\frac{1}{t_{f}}\left(1-\frac{\omega_{d}^{2}}{\omega_{n}^{2}}\right) & \frac{1}{t_{f}}\left(-1+\frac{\omega_{d}^{2}}{\omega_{n}^{2}}\right) & \frac{2}{t_{f}} \cdot\left(\frac{\xi_{d} \cdot \omega_{d}}{\omega_{n}^{2}}-\frac{\xi_{n}}{\omega_{n}}\right) & -\frac{1}{t_{f}}
\end{array}\right] \\
\mathbf{B}_{1}=\left[\begin{array}{cccc}
\frac{1}{C} & 0 & 0 & 0
\end{array}\right]^{t}
\end{gathered}
$$

Nota-se que os elementos da matriz A são fortemente dependentes do ângulo de disparo. No entanto, como o ângulo de disparo é variável atuante no controle, é interessante que esta variável se torne uma entrada no sistema. Assim, a partir da linearização das equações de estado com relação ao ângulo de disparo, têm-se o novo sistema em (19).

$$
\dot{\mathbf{x}}=\mathbf{A} \cdot \mathbf{x}+\mathbf{B}_{1} \cdot i+\mathbf{B}_{2} \cdot \alpha
$$

Onde:

$$
\mathbf{B}_{2}=\left[\begin{array}{llll}
b_{21} & b_{22} & b_{23} & b_{24}
\end{array}\right]^{t}
$$

Os coeficientes de $\mathbf{B}_{2}$ são determinados a partir da linearização das equações de estado em torno de um ponto de operação. Para o sistema CA os valores de regime permanente senoidal, ou do ponto de operação, são considerados para a condição descrita em (21).

$$
\frac{d()}{d t}=s=j . \omega_{1}
$$

Aplicando-se (21) em (16) determina-se os valores do ponto de operação (PO) das variáveis de estado. Tais valores em módulo são apresentados de (22) a (25).

$$
\begin{gathered}
X_{1}=\frac{I}{\omega_{1} \cdot C} \\
X_{2}=\frac{V_{1}}{\sqrt{\left(\left(\frac{\omega_{1}}{\omega_{d}}\right)^{2}+1\right)^{2}+\left(\frac{\xi_{d}}{\omega_{d}^{2}} \cdot \omega_{1}\right)^{2}}} \\
X_{3}=\omega_{1} \cdot X_{2} \\
X_{4}=V_{C}
\end{gathered}
$$

Uma vez conhecido o ponto de operação do sistema, podem-se determinar os coeficientes de $\mathbf{B}_{\mathbf{2}} \mathrm{em}$ (26) a (29).

$$
\begin{aligned}
& b_{21}=\left.\frac{d \dot{x}_{1}}{d \alpha}\right|_{P O} \\
& b_{22}=\left.\frac{d \dot{x}_{2}}{d \alpha}\right|_{P O}
\end{aligned}
$$




$$
\begin{aligned}
& b_{23}=\left.\frac{d \dot{x}_{3}}{d \alpha}\right|_{P O} \\
& b_{24}=\left.\frac{d \dot{x}_{4}}{d \alpha}\right|_{P O}
\end{aligned}
$$

Aplicando a transformada de Laplace em (19), considerando nulas as variações na corrente de linha, tem-se em (30) as funções de transferência que relacionam as variáveis de estado com a entrada de controle $(\alpha)$

$$
\left[\frac{X(s)}{\alpha(s)}\right]=\left[\begin{array}{c}
0 \\
\frac{b_{23}}{s^{2}+2 \cdot \xi_{d} \cdot \omega_{d} \cdot s+\omega_{d}^{2}} \\
\frac{b_{23} \cdot s}{s^{2}+2 \cdot \xi_{d} \cdot \omega_{d} \cdot s+\omega_{d}{ }^{2}} \\
\frac{b_{22} \cdot\left(2 \cdot \xi_{d} \cdot \omega_{d} \cdot s-2 \cdot \xi_{n} \cdot \omega_{n} \cdot s-\omega_{n}^{2}+\omega_{d}^{2}\right)}{\omega_{n}^{2} \cdot\left(s^{2}+2 \cdot \xi_{d} \cdot \omega_{d} \cdot s+\omega_{d}^{2}\right) \cdot\left(s \cdot t_{f}+1\right)}+\frac{b_{24}}{s+\frac{1}{t_{f}}}
\end{array}\right]
$$

A função de transferência que nos interessa para o projeto do controlador é a que representa a relação entre as variações da tensão no capacitor e as variações no ângulo de disparo. Tal função está representada na última linha da matriz em (30). Considerando $\mathrm{t}_{\mathrm{f}}$ muito pequeno e rearranjando os termos, a função de transferência pode ser apresentada no formato indicado em (31).

$$
G_{v \alpha}(s)=G_{v \alpha 0} \cdot \frac{\left(\frac{s}{\omega_{z}}+1\right)}{\left(\frac{s}{1 / t_{f}}+1\right) \cdot\left(\frac{s^{2}}{\omega_{d}^{2}}+\frac{2 \cdot \xi_{d} \cdot s}{\omega_{d}}+1\right)}
$$

Onde:

$$
\begin{gathered}
G_{v \alpha 0}=b_{32 \cdot} \cdot\left(\frac{1}{\omega_{n}^{2}}-\frac{1}{\omega_{d}^{2}}\right) \\
\omega_{z}=\frac{1}{2} \cdot\left(\frac{\omega_{d}^{2}-\omega_{n}^{2}}{\xi_{d} \cdot \omega_{d}-\xi_{n} \cdot \omega_{n}}\right) \\
b_{32}=\left(\frac{4}{\pi \cdot \sqrt{L_{r c t} \cdot C}}\right) \cdot\left(\frac{\pi-2 \cdot \alpha}{\pi \cdot \sqrt{L_{r c t} \cdot C}}\right) \cdot\left(X_{2}-X_{1}\right)+ \\
\left(\frac{X_{3} \cdot 0,76}{\pi \cdot \sqrt{L_{r c t} \cdot C}}\right) \cdot((\pi-2 \cdot \alpha) \cdot \operatorname{sen}(\alpha)+1,52 \cdot \cos (\alpha))
\end{gathered}
$$

Para que a magnitude da tensão do capacitor siga o valor de referência, com erro nulo em regime permanente, a magnitude do ganho da malha de tensão $\mathrm{T}(\mathrm{s})$ deve ser elevada nas baixas frequências. Tal função de transferência é representada em (35) e leva em consideração os efeitos do controlador.

$$
T(s)=G_{C}(s) \cdot G_{v \alpha}(s)
$$

No entanto, um ganho de malha elevado pode levar o sistema à instabilidade ocasionada pela inversão de fase na malha de realimentação. Nesse sentido, a fase da função de transferência de $\mathrm{T}(\mathrm{s})$ não deve ultrapassar -180 graus na frequência de cruzamento, respeitando assim o critério de margem de fase (MF) para a estabilidade de sistemas.
A frequência de cruzamento de $\mathrm{T}(\mathrm{s})$ determina a largura de banda passante do sistema em malha fechada da saída com relação à referência, que influencia diretamente na velocidade e na resposta dinâmica do sistema de controle.

\section{Resultados de Simulação}

Os resultados de simulação apresentados nesta seção subsidiarão a construção de um protótipo do TCSC em bancada laboratorial. A tabela 1 resume as características da linha simulada.

Tabela 1. Característica da linha (valores por fase)

\begin{tabular}{ccc}
\hline Simbologia & Descrição & Valores \\
\hline $\mathrm{V}_{\mathrm{S}}$ & Tensão da S/E & $127 \mathrm{~V}$ \\
\hline $\mathrm{f}_{1}$ & Frequência fundamental & $60 \mathrm{~Hz}$ \\
\hline $\mathrm{R}$ & Resistência da linha & $0,67 \Omega$ \\
\hline $\mathrm{X}_{\mathrm{L}}$ & Reatância de linha & $2,00 \Omega$ \\
\hline $\mathrm{P}$ & Potência ativa & $1000 \mathrm{~W}$ \\
\hline $\mathrm{Q}$ & Potência reativa & $340 \mathrm{Var}$ \\
\hline $\mathrm{Irms}$ & Corrente eficaz de linha & $9,64 \mathrm{~A}$ \\
\hline
\end{tabular}

$\mathrm{Na}$ tabela 2 são apresentadas as especificações do TCSC.

Tabela 2. Especificações do TCSC

\begin{tabular}{ccc}
\hline Simbologia & Descrição & Valores \\
\hline$\alpha_{\mathrm{r}}$ & $\begin{array}{c}\text { Ângulo de disparo para } \\
\text { ressonância }\end{array}$ & $45^{\circ}$ \\
\hline $\mathrm{X}_{\mathrm{C}}$ & Reatância capacitiva $\left(\alpha=90^{\circ}\right)$ & $1,2 \Omega$ \\
\hline $\mathrm{L}_{\mathrm{RCT}}$ & Indutância do RCT & $795,77 \mu \mathrm{H}$ \\
\hline $\mathrm{C}$ & Capacitância fixa do TCSC & $2,2 \mathrm{mF}$ \\
\hline $\mathrm{K}_{\mathrm{C}}$ & Faixa de compensação reativa & $60 \%$ a $200 \%$ \\
capacitiva & $1,2 \Omega$ a $4,0 \Omega$ \\
\hline
\end{tabular}

A curva de reatância característica do TCSC especificado é apresentada na Figura 4.

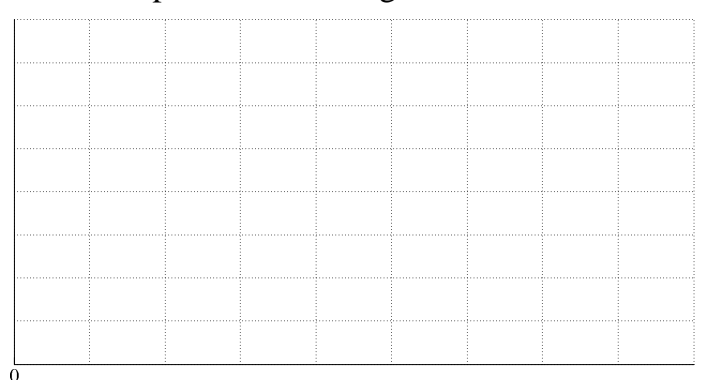


dos na Figura 5 considerando-se três pontos de operação distintos pertencentes à PO1. 


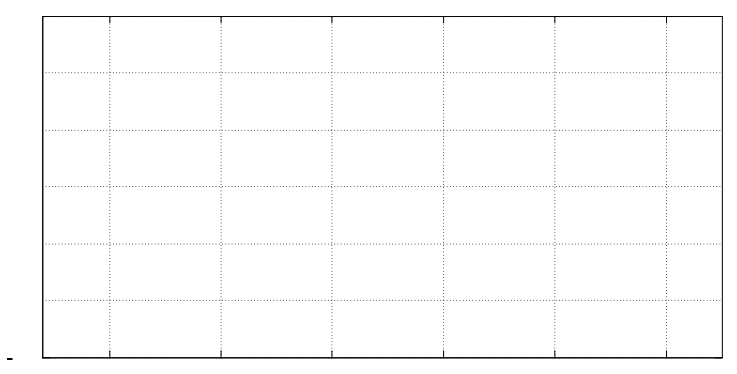

